\title{
Article \\ Enhanced Sensitivity of FeGa Thin-Film Coated SAW Current Sensor
}

\author{
Yuan Sun ${ }^{1,2}$, Yana Jia ${ }^{1}$, Yufeng Zhang ${ }^{1}$, Lina Cheng ${ }^{1}$, Yong Liang ${ }^{1}$ and Wen Wang ${ }^{1,2, *}$ \\ 1 Institute of Acoustics, Chinese Academy of Sciences, Beijing 100190, China; sunyuan@mail.ioa.ac.cn (Y.S.); \\ jiayana225@163.com (Y.J.); 17701353856@163.com (Y.Z.); chenglina@mail.ioa.ac.cn (L.C.); \\ liangyong@mail.ioa.ac.cn (Y.L.) \\ 2 School of Electronic, Electrical and Communication Engineering, University of Chinese Academy of Sciences, \\ Beijing 100049, China \\ * Correspondence: wangwenwq@mail.ioa.ac.cn
}

Citation: Sun, Y.; Jia, Y.; Zhang, Y.;

Cheng, L.; Liang, Y.; Wang, W.

Enhanced Sensitivity of FeGa

Thin-Film Coated SAW Current

Sensor. Appl. Sci. 2021, 11, 11726.

https://doi.org/10.3390/

app112411726

Academic Editors: Junseop Lee and Hyeonseok Yoon

Received: 7 November 2021

Accepted: 8 December 2021

Published: 10 December 2021

Publisher's Note: MDPI stays neutral with regard to jurisdictional claims in published maps and institutional affiliations.

Copyright: () 2021 by the authors. Licensee MDPI, Basel, Switzerland. This article is an open access article distributed under the terms and conditions of the Creative Commons Attribution (CC BY) license (https:// creativecommons.org/licenses/by/ $4.0 /)$.

\begin{abstract}
A surface acoustic wave (SAW) device is proposed for sensing current by employing the patterned FeGa thin film as the sensitive interface. The layered media structure of $\mathrm{FeGa} / \mathrm{SiO}_{2} / \mathrm{LiNbO}_{3}$ was established to reveal the working principle of the sensors, and an SAW chip patterned by

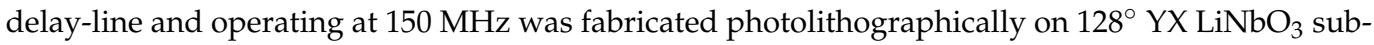
strate. The FeGa thin film with a larger magnetostrictive coefficient was sputtered onto the acoustic propagation path of the SAW chip to build the sensing device. The prepared device was connected into the differential oscillation loop to construct the current sensor. The FeGa thin film produces magnetostrictive strain and so-called $\Delta E$ effect at the magnetic field generated by the applied current, which modulates the SAW propagation velocity accordingly. The differential frequency signal was collected to characterize the measurand. Larger sensitivity of $37.9 \mathrm{kHz} / \mathrm{A}$, low hysteresis error of $0.81 \%$, excellent repeatability and stability were achieved in the experiments from the developed sensing device.
\end{abstract}

Keywords: surface acoustic wave; current sensor; FeGa thin film; magnetostrictive strain; $\Delta E$ effect

\section{Introduction}

Current sensors are widely used in current monitoring applications in smart grid line testing, metallurgical and power supplies, rail transit safety warnings and rescue, and power relay protection in industrial automation [1]. Among the available sensing technologies, the surface acoustic wave (SAW) current sensor features fast response, simple structure, low cost, excellent resistance to interference, low power consumption, and long service life [2-4]. Especially, it can realize wireless and passive measurement means to improve system security [5-7]. The specialized current sensing prototype employing the magnetoresistance effect was proposed firstly by Reindl et al. The obtained current resolution was approximately $5 \%$ of full scale (-800 A 800 A) [6]. Another typical SAW current sensing device is built by depositing magnetostrictive thin film along the acoustic propagation path of the SAW chip. The magnetostrictive thin film produces magnetostrictive strain and so-called $\Delta E$ effect at the magnetic field generated by the applied current, which modulates the SAW propagation velocity accordingly. Then the corresponding shifts in oscillation frequency are collected to evaluate the applied current information [1,8,9].

Since the pioneering work was conducted by Ganguly et al. in 1975 [10], the SAW devices for sensing current/magnetic field have attracted more interests because of their unique advantages, and meaningful results were reported from some prototypes. Using the FeCo thin film as the sensitive interface, the SAW current sensor prototype was constructed successfully, a larger sensitivity of $16.6 \mathrm{kHz} / \mathrm{A}$ was achieved, and the patterned design was considered to improve the hysteresis error [1,8,9]. A similar structure was also proposed by Tong et al.; the current sensitivity of $10.7 \mathrm{kHz} / \mathrm{A}$ was achieved by using the FeNi as the 
sensing interface [11]. Kadota et al. developed a SAW sensing chip constructed using a magnetostrictive Ni electrode on ST-cut $90^{\circ} \mathrm{X}$ quartz substrate [12]. Zhou et al. obtained a maximum SAW velocity shift close to $20 \%$ from a multilayered sensing structure of $\mathrm{TbCo}_{2} / \mathrm{FeCo}$ for the shear horizontal wave as a ratio close to 1 between magneto-elastic film thickness and wavelength [13]. Fahim et al. proposed a SAW magnetic sensor using Polyvinyl Alcohol (PVA) bound magnetostrictive nanopowder thin film, and a sensor response of up to $678.05 \mathrm{kHz}$ was obtained towards a magnetic field of $120 \mathrm{mT}$ [14]. Dong et al. demonstrated that laminate composed of longitudinally magnetized magnetostrictive Terfenol- $\mathrm{D}$ and a transversely poled piezoelectric $\mathrm{Pb}\left(\mathrm{Mg}_{1 / 3} \mathrm{Nb}_{2 / 3}\right) \mathrm{O}_{3}-\mathrm{PbTiO}_{3}$ crystal offered extremely high magnetic field sensitivity of $10^{-11} \mathrm{~T}$ [15]. Schell et al. analyzed the influence of the deposition process and heat treatment on the performance of devices to improve the limits of detection [16]. Sun et al. deposited the FeGaB film on the AlN piezoelectric materials, which enabled a measurement range of up to $300 \mathrm{pT}$ in the presence of a DCbiased magnetic field [17]. Taking the magnetostriction effect, $\Delta E$ effect, and the third-order material constants into account, Yang et al. investigated the sensing mechanism of SAW magnetic field sensors [18], and they also explored a grooved sensing surface structure to improve sensitivity.

Obviously, the performance of an SAW-based current/magnetic sensor is significantly determined by the magnetostrictive coatings [19]. Terfenol-D features a larger magnetostrictive coefficient. Therefore, very high magnetic sensitivity will be expected. However, an easily oxidized nature prevents its application. Meanwhile, FeGa features lower coercivity, higher Curie temperature, lower cost, and excellent mechanical properties [20], and maximum magnetostriction coefficient is up to $400 \mathrm{ppm}$ [21], which is much larger than that of $\mathrm{Fe}, \mathrm{Co}, \mathrm{Ni}$ and their alloys [22,23]. In addition, the FeGa thin film applied for microsensor and microsystem integration is conducive to the miniaturization and intelligence of the sensor, and it significantly improves the performance of the device.

In this contribution, a new design of SAW device for sensing current is proposed by employing the patterned FeGa thin film as the sensitive interface, as depicted in Figure 1. A SAW chip with a delay-line pattern was fabricated photolithographically on $128^{\circ}$ YX $\mathrm{LiNbO}_{3}$ substrate to operate at $150 \mathrm{MHz}$. To improve the sensitivity and suppress the hysteresis error, the patterned design was performed to the FeGa thin film. The proposed current sensing device was built by sputtering the FeGa thin film to the SAW chip and characterized by connecting it into the differential oscillation loops. Larger current sensitivity, low hysteresis error, excellent repeatability and stability were achieved experimentally.

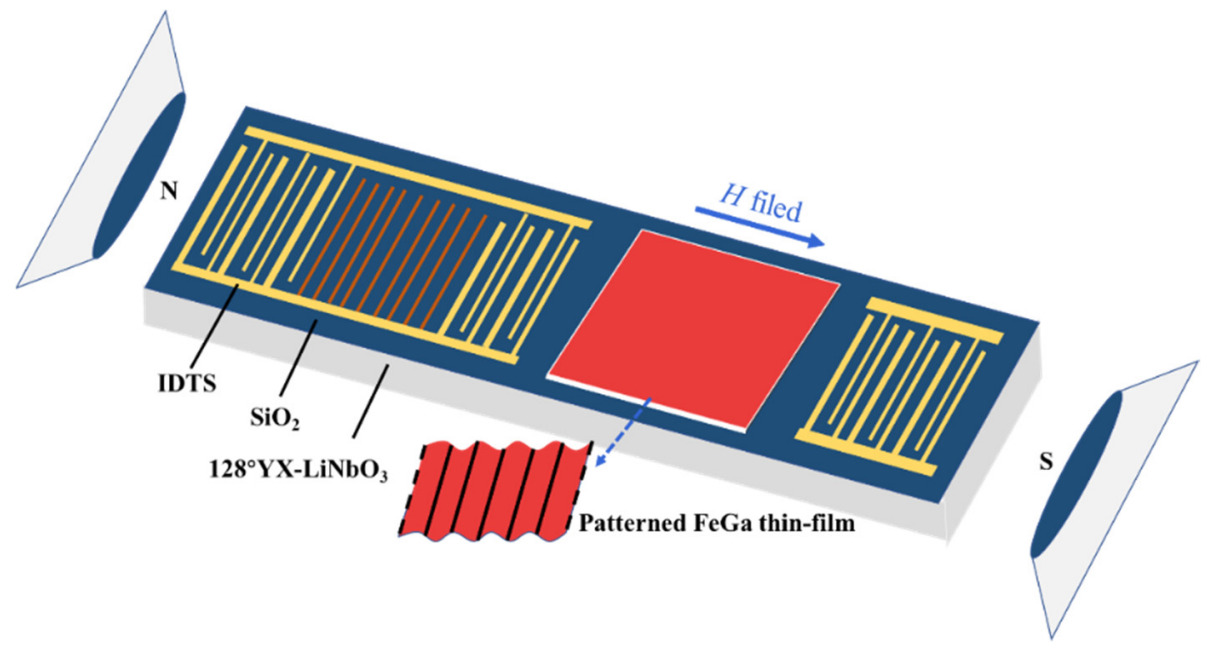

Figure 1. The scheme of patterned FeGa thin film coated SAW sensing device.

\section{Working Principle}

Under the magnetic field generated by the applied current, the FeGa film produces magnetostrictive strain and so-called $\Delta E$ effect, which modulates the SAW propagation. 
A layered media structure of $\mathrm{FeGa} / \mathrm{SiO}_{2} / \mathrm{LiNbO}_{3}$ is proposed to demonstrate the sensing mechanism, as shown in Figure 2.

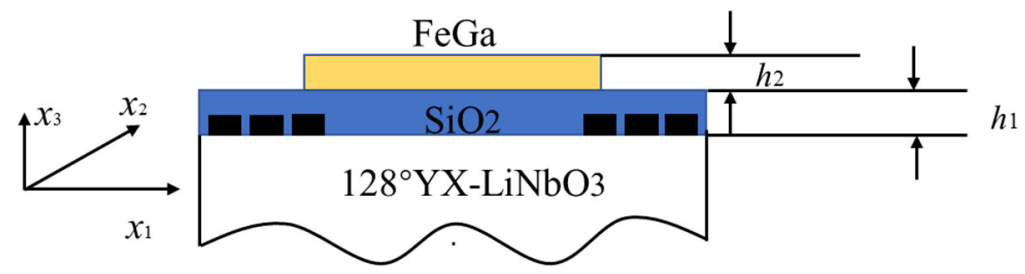

Figure 2. The layered media model $\left(\mathrm{FeGa} / \mathrm{SiO}_{2} / \mathrm{LiNbO}_{3}\right)$.

(1) In the piezoelectric media

The constitutive wave motion equations in a piezoelectric $\mathrm{LiNbO}_{3}$ can be expressed as

$$
\left\{\begin{array}{c}
\rho_{S} \frac{\partial^{2} u_{i}^{I}}{\partial t^{2}}-c_{j k l}^{I} \frac{\partial^{2} u_{k}^{I}}{\partial x_{l} \partial x_{j}}-e_{k i j} \frac{\partial^{2} \Phi}{\partial x_{k} \partial x_{j}}=0 \\
e_{j k l} \frac{\partial^{2} u_{k}^{I} \partial x_{j}}{\partial x_{j}}-\varepsilon_{j k}^{S} \frac{\partial^{2} \Phi}{\partial x_{k} \partial x_{j}}=0
\end{array}\right.
$$

where Einstein's summation rule is used. The indices $i, j, k, l=1,2,3$, and $c^{I}, e, \varepsilon, \rho_{S}$ stand for the elastic, piezoelectric, dielectric constants and the mass density of the piezoelectric substrate, respectively. $u^{I}$ denotes the mechanical displacements, and $\Phi$ denotes the electric potential.

(2) In the $\mathrm{SiO}_{2}$ media,

The $\mathrm{SiO}_{2}$ media is considered as the isotropic and nonpiezoelectric media. Then, the acoustic wave equation can be written as

$$
c_{i j k l}^{I I} \frac{\partial^{2} u_{k}^{I I}}{\partial x_{l} \partial x_{j}}=\rho_{a} \frac{\partial^{2} u_{i}^{I I}}{\partial t^{2}}
$$

where $u^{I I}, c^{I I}$ and $\rho_{a}$ are the mechanical displacements, stiffness constants and density of the $\mathrm{SiO}_{2}$ film, respectively.

(3) In the FeGa media,

The acoustic wave equation in the FeGa media can also be written as

$$
c_{i j k l}^{I I I} \frac{\partial^{2} u_{k}^{I I I}}{\partial x_{l} \partial x_{j}}=\rho_{b} \frac{\partial^{2} u_{i}^{I I I}}{\partial t^{2}}
$$

where $u^{I I I}, c^{I I I}$ and $\rho_{b}$ are the mechanical displacements, the stiffness constants and density of the FeGa film, respectively.

(4) Magnetostrictive strain of the FeGa film

Under the magnetic field generated by the applied current, the FeGa film produces magnetostrictive strain, which leads to changes in thickness $h$ and density $\rho_{f}$ of FeGa film.

$$
\left\{\begin{array}{c}
h=h_{0}\left(1-\frac{\lambda}{2}\right) \\
\rho_{f}=\rho_{0} /\left[(1+\lambda) \times\left(1-\frac{\lambda}{2}\right) \times\left(1-\frac{\lambda}{2}\right)\right]
\end{array}\right.
$$

where $h_{0}$ and $\rho_{0}$ are the thickness and density of unperturbed FeGa film, respectively, and $\lambda$ is the magnetostrictive coefficient of the FeGa film.

(5) $\Delta E$ effect on the FeGa film 
The external magnetic field will also modulate the Young's modulus $(E)$ of the FeGa film, which is so-called $\Delta E$ effect, and the corresponding elastic coefficients in FeGa film are expressed by the perturbed Young's modulus $E^{\prime}$ as

$$
\left\{\begin{array}{c}
c_{11}=\frac{E^{\prime}(1-u)}{(1+u)(1-2 u)} \\
c_{12}=\frac{E^{\prime} u}{(1+u)(1-2 u)} \\
c_{44}=\frac{c_{11}-c_{12}}{2}=\frac{E^{\prime}(1-2 u)}{2(1+u)(1-2 u)}
\end{array}\right.
$$

where $u$ is the Poisson's ratio.

The relationship between the electromagnetic field intensity $(H)$ and current $(A)$ can be described by the Biot-Savart law [24],

$$
H=\frac{\mu_{0} I}{4 \pi l}
$$

where $\mu_{0}$ denotes the permeability in vacuum. $l$ expresses the distance between the sensor chip and wires, which is set to $1 \mathrm{~cm}$.

According to the Formulas (1)-(3), the solution of each layer media can be obtained. Then, combined with the mechanics and electrical boundary conditions, the effective surface permittivity method can be used to obtain the speed of SAW. Under the magnetic field generated by the applied current, according to the Formulas (4)-(5), the FeGa film produces magnetostrictive strain and so-called $\Delta E$ effect, which modulates the SAW propagation.

The measured magnetostriction curve characteristic of FeGa is shown in Figure 3a. The simulation was done to predict the sensitivity of the proposed sensor. The parameters of $\mathrm{LiNbO}_{3}$ and $\mathrm{SiO}_{2}$ used in the simulation were consistent with the literature [11]. The mechanics and electrical boundary conditions were also the same as the literature [11]. The film thickness was set to $500 \mathrm{~nm}$, and the aspect ratio was set to 1:1. The sensitivity of the proposed current sensor is calculated as $\sim 20.3 \mathrm{kHz} / \mathrm{A}$, as shown in Figure 3b. Compared with the sensitivity of $\sim 11 \mathrm{kHz} / \mathrm{A}$ mentioned in the literature [11], the sensitivity of the proposed sensor is increased by $\sim 9 \mathrm{kHz} / \mathrm{A}$. The results reveal that the FeGa film can effectively improve sensor performance.
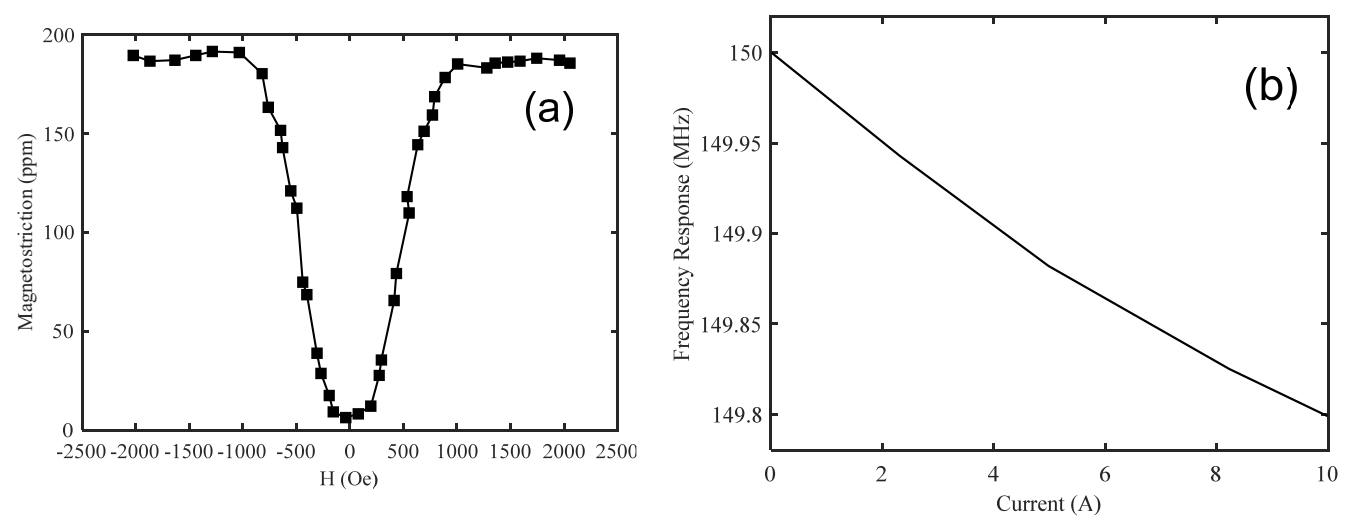

Figure 3. (a) The magnetostriction curve characteristic of $\mathrm{FeGa}$; (b) the predicted sensitivity of the $150 \mathrm{MHz}$ SAW-based current sensor.

\section{Technical Realizations}

\subsection{SAW Devices}

$\mathrm{LiNbO}_{3}$ piezoelectric crystal features a larger electromechanical coupling coefficient $\left(K^{2}\right)$, which benefits the reduction in insertion loss of SAW device. Therefore, $128^{\circ} \mathrm{YX}$ $\mathrm{LiNbO}_{3}$ piezoelectric crystal with large $K^{2}(5.5 \%)$ was chosen as the piezoelectric substrate of the sensing chip with a delay-line pattern, and FeGa thin film was sputtered to the SAW propagation path between the two photolithographically defined $300 \mathrm{~nm} \mathrm{Al}$ transducers separated by a path length of $\sim 2 \mathrm{~mm}$. Single-phase unidirectional transducers (SPUDTs) 
confining the acoustic energy predominantly in one direction on the piezoelectric substrate surface were used to form the transducers to reduce the insertion loss [1]. The operation frequency was designed to $150 \mathrm{MHz}$, and the corresponding wavelength $\lambda$ was $25.84 \mu \mathrm{m}$. The electrode widths in SPUDTs were $3.23 \mu \mathrm{m}(\lambda / 8)$ and $6.46 \mu \mathrm{m}(\lambda / 8)$. The lengths of the input transducer and output transducer were designed to be $130 \lambda$ and $40 \lambda$, respectively. In addition, a comb structure was designed for the transducers to eliminate other unwanted vibration frequencies to achieve a single oscillation mode [25]. After the $\mathrm{Al}$ electrodes preparation, a $30 \mathrm{~nm} \mathrm{SiO} 2$ thin film was covered on the transducers by PECVD to protect the electrodes in FeGa deposition.

\subsection{Preparation of $\mathrm{FeGa}$ Film}

Then, the FeGa thin film was deposited onto the acoustic propagation path of the developed SAW chip to build the sensing device by employing the lift-off process. The corresponding details are described in Figure 4. Firstly, the photoresist was deposited on the prepared SAW device. Then, through a photolithography mask, the photoresist was removed in the areas, where the FeGa thin film was to be located; then, the FeGa thin film was sputtered onto the surface of the SAW chip using radio-frequency magnetron sputtering. The sputtering conditions for the base pressure, sputtering power and targetsubstrate distance were $1.5 \times 10^{-5} \mathrm{~Pa}, 100 \mathrm{~W}$, and $60 \mathrm{~mm}$, respectively. The Ar gas was used as the sputtering gas, and corresponding pressure was set to $1 \mathrm{~Pa}$. After removed the remaining photoresist, the FeGa thin film with various patterns (dot, grating, and membrane) was formed on the SAW chip. Here, the strip width in each FeGa grating was set to $3 \lambda$, and the corresponding grating spacing was set to $4 \lambda$. Similarly, the length width of each FeGa dot was set to be $3 \lambda \times 3 \lambda$, and dot spacing in each direction was set to $4 \lambda$. The developed SAW sensing devices with various FeGa thin-film patterns were pictured in Figure 5a-c.

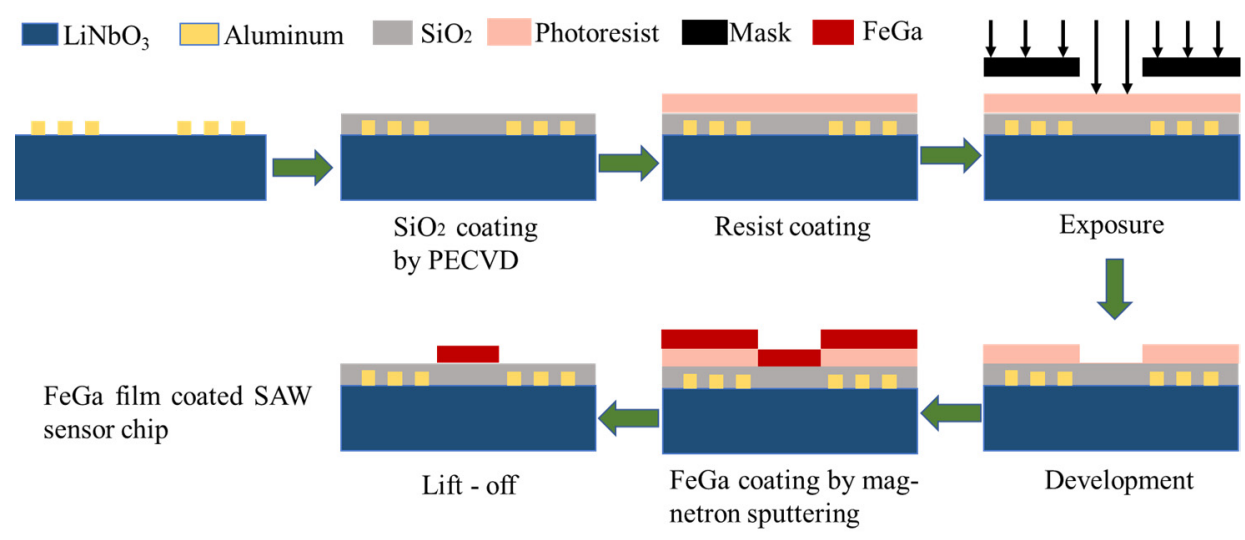

Figure 4. The fabrication process of FeGa film.

Then, the deposited FeGa thin film was characterized by using the scanning electron microscope (SEM) and 2D atomic force microscope (AFM), as shown in Figure 5d,e. It can be seen that the surface of FeGa film was relatively smooth, and the distribution was dense and uniform. The corresponding surface average roughness $(\mathrm{Ra})$ was about $1.56 \mathrm{~nm}$, and the maximum height difference on the surface $(\mathrm{P}-\mathrm{V})$ was $\sim 15.69 \mathrm{~nm}$.

Using the network analyzer, we characterized the developed sensing devices as shown in Figure 5f. Among them, the working frequency of the sensing devices with various FeGa patterns (dot, grating, and membranous) were measured as $150.4 \mathrm{MHz}, 149.7 \mathrm{MHz}$, and $149.4 \mathrm{MHz}$, respectively, and their corresponding insertion losses were both less than $10 \mathrm{~dB}$. The deviation in operation frequency stems from the manufacturing error, and there are almost no unacceptable effects in sensing performance. 


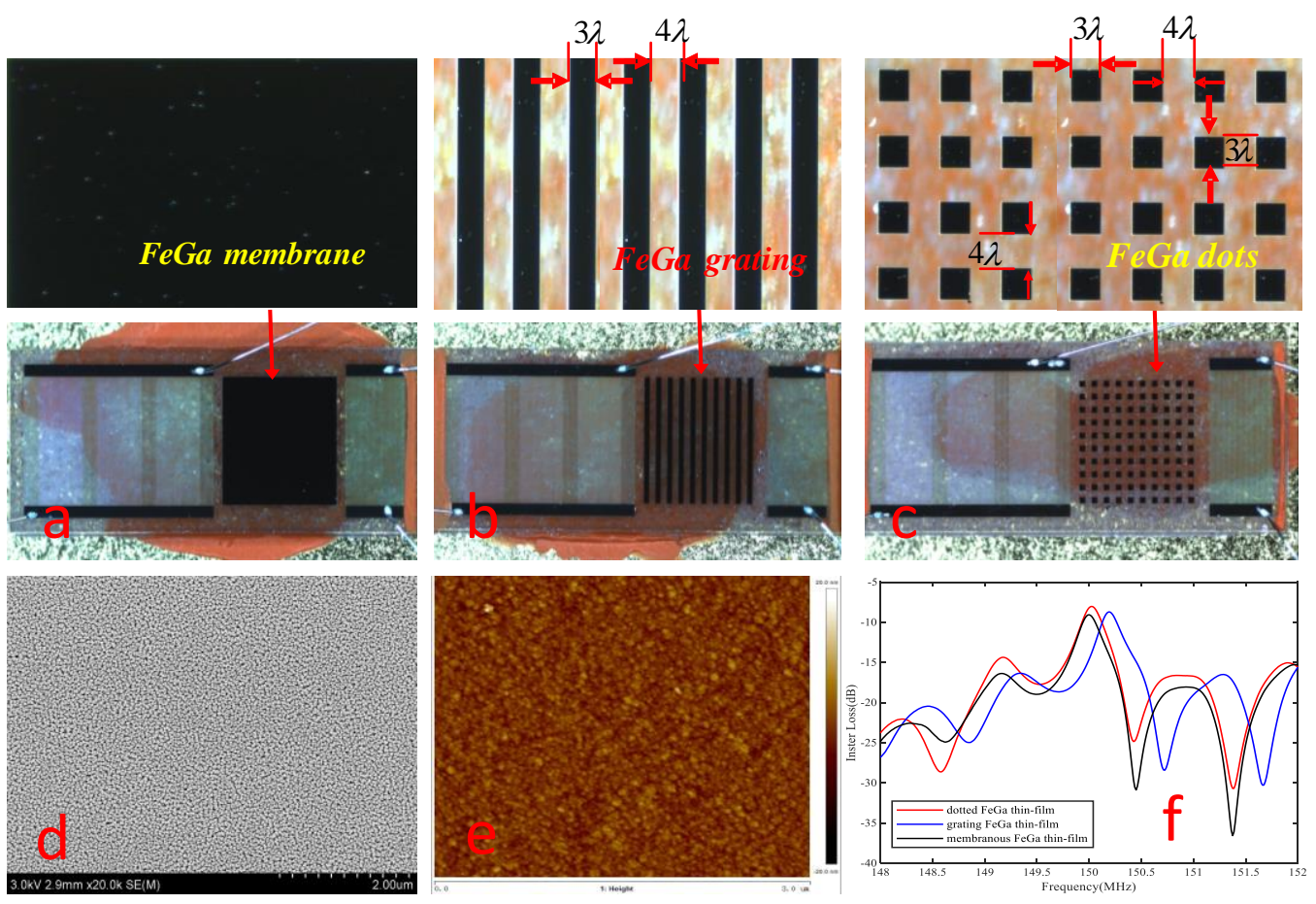

Figure 5. Optica pictures of developed sensing device coated with (a) FeGa membranous, (b) FeGa grating, (c) FeGa dots, (d) SEM view, and (e) AFM picture of the FeGa thin film, and (f) measured frequency characteristic of the fabricated sensing devices.

\subsection{Differential Oscillator}

In this work, the oscillation loop was employed to generate the SAW chip, and the differential structure was also used to eliminate the external temperature effect, vibration noise, and magnetic noise [26-28]. The FeGa thin film coated device as the sensing chip and the naked one as the reference chip were packaged into the same metal base, and connected into the differential oscillation loop made of discrete elements as an amplifier, phase shifter, mixer, and so on (Figure 6a), and the mixed oscillation frequency signal was collected by using the frequency counter as the sensing signal.
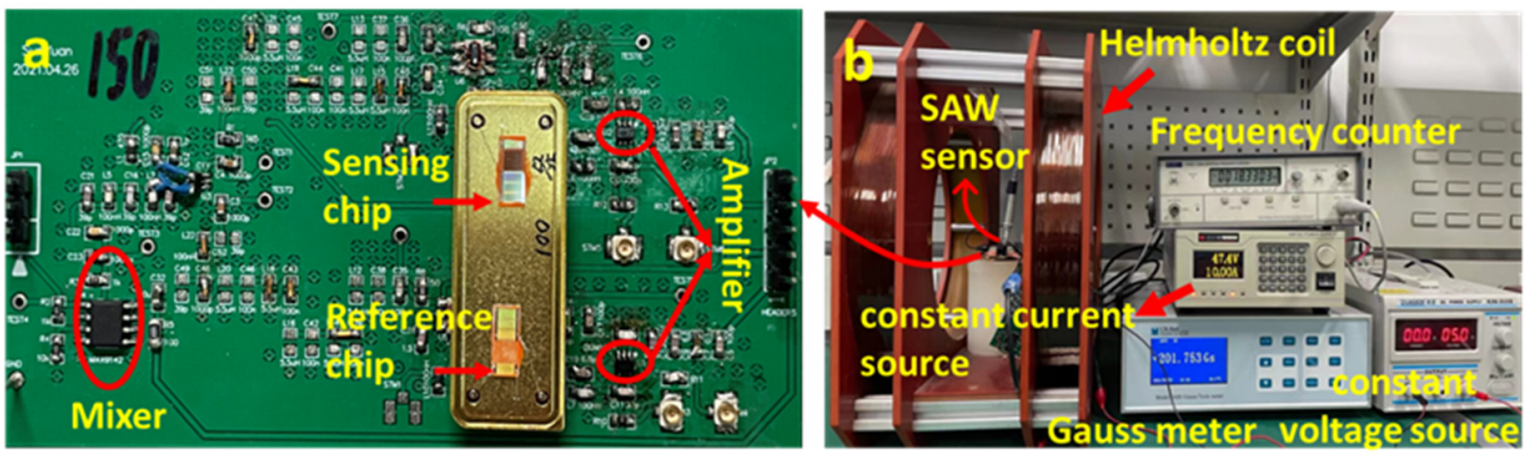

Figure 6. (a)The view of oscillation circuit board with sensor chip; (b) the experimental setup for characterizing the SAW sensors.

\section{Sensor Experiments and Discussions}

\subsection{Experimental Setup}

The experimental setup for characterizing the developed SAW sensor is described in Figure $6 \mathrm{~b}$, which is composed of a Helmholtz coil system with measure range of $0 \sim 10 \mathrm{~A}$, Gaussmeter, SAW sensor, frequency counter, constant current source, and constant voltage source. By varying the coil current generated by the constant current source, the Helmholtz 
coil can create a varying magnetic field. The differential oscillation loop is supplied with a voltage of $+5 \mathrm{~V}$ provided by the constant voltage source. The sensor signal is collected by the frequency counter at 60 points per minute and is plotted by the PC in real time. During the test, the SAW device was exposed to the magnetic field, and the differential oscillation loop was wrapped with aluminum foil so as to reduce the influence of the magnetic field on the circuit.

\subsection{Sensor Performance Evaluation}

\subsubsection{Sensitivity Evaluation}

First, the sensitivity of the prepared sensor was evaluated by measuring the sensing response towards increasing current values from 0 to $10 \mathrm{~A}$ at room temperature $\left(25^{\circ} \mathrm{C}\right)$ using the experimental setup described in Figure $6 \mathrm{~b}$. The effect from FeGa film thickness on sensitivity was demonstrated by measuring the sensitivity of developed sensors with various FeGa film thicknesses $(300 \mathrm{~nm}, 500 \mathrm{~nm}$, and $700 \mathrm{~nm}$ ), as shown in Figure 7a. Obviously, the sensitivity increases with increasing thickness of FeGa film. This can be explained by the following formula [29]:

$$
\frac{\Delta V}{V}=-f h \sum_{i=1}^{3} c_{i}\left(\rho-\frac{E^{(i)}}{V^{2}}\right)
$$

where $f, h, c_{i}$ and $E^{(i)}$ are the operation frequency of SAW device, FeGa film thickness, coupling parameter relating to the piezoelectric substrate, and Young's modulus of the FeGa film, respectively. It can be seen that the acoustic velocity is proportional to the film thickness when other parameters are fixed. Therefore, increasing the FeGa film thickness will improve the sensitivity. However, a turning point occurred when thicker FeGa film over $700 \mathrm{~nm}$ was applied. This is because the magnetic domain wall gradually changed from Neel wall to Bloch wall with the increase in the film thickness, resulting in the degradation of the film soft magnetic properties. The result indicates that too thick of a FeGa film will reduce the sensitivity. Hence, $500 \mathrm{~nm}$ FeGa film was chosen to construct the sensing devices in this work.
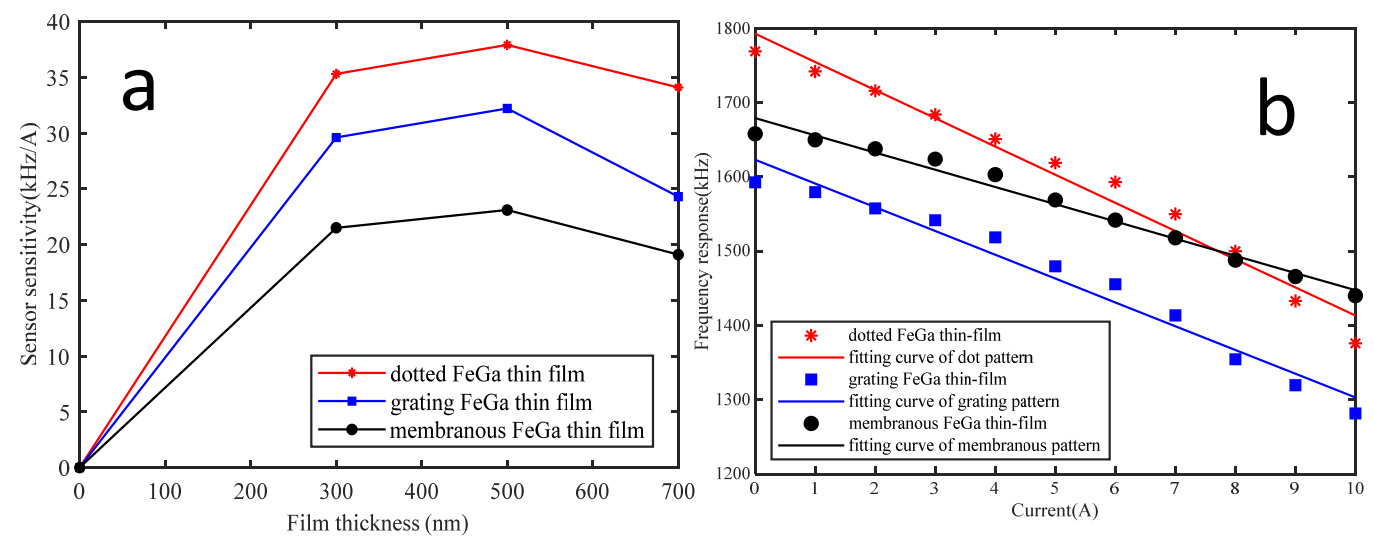

Figure 7. (a) The effects of film thickness on the sensor sensitivity; (b) the frequency response of current sensor with a $500 \mathrm{~nm}$ thick film.

Figure $7 \mathrm{~b}$ shows the linear response of the proposed sensor coated with $500 \mathrm{~nm}$ patterned FeGa thin film. The concluded sensitivities of $37.9 \mathrm{kHz} / \mathrm{A}, 32.2 \mathrm{kHz} / \mathrm{A}, 23.1 \mathrm{kHz} / \mathrm{A}$ were obtained from the sensing devices coated with dotted, grating, and membranous FeGa thin films, respectively. The sensitivity of membranous FeGa thin film coated device approximately agreed with the predicted value. The largest sensitivity was achieved from the dotted-FeGa thin film coated device, which is the result of enlarged magnetostrictive properties and reduced coercivity in the dot-film structure [9]. 


\subsubsection{Repeatability Test}

Then, the repeatability of the $500 \mathrm{~nm}$ FeGa thin film coated SAW sensing device was evaluated as shown in Figure 8, which shows response profiles obtained from four consecutive $5 \mathrm{~s}$ on-off exposed to $10 \mathrm{~A}$ current at room temperature $\left(25^{\circ} \mathrm{C}\right)$. To evaluate the repeatability, a statistical analysis was performed to the measured results. Using the sensor response towards applied current of $10 \mathrm{~A}$ as a sample set (Figure 8), we evaluated repeatability by calculating the corresponding standard deviation. Usually, the standard deviation is defined by

$$
\sigma=\sqrt{\sum_{i=1}^{N}\left(x_{i}-\bar{x}\right) /(N-1)}
$$

where $x_{i}, \bar{x}$, and $N$ are the $i$ th measurement, the average of the measurements, and the number of measurements, respectively. Hence, the standard deviations of $0.99,0.71$, and 1.13 were calculated from the sensing devices coated with dotted, grating, and membranous FeGa thin film, respectively. It can be seen that excellent reproducible runs were obtained from the prepared sensing device with various FeGa patterns. Moreover, when the current was switched from $0 \mathrm{~A}$ to $10 \mathrm{~A}$, the frequency response of the three sensors dropped rapidly to a steady state, which means a very fast response was achieved. Moreover, sensor responses over $200 \mathrm{kHz}$ towards $10 \mathrm{~A}$ were obtained from the sensing devices. The largest response up to $393 \mathrm{kHz}$ was achieved from the sensing device coated with dotted $\mathrm{FeGa}$ thin film owing to its enlarging magnetostrictive properties [9].

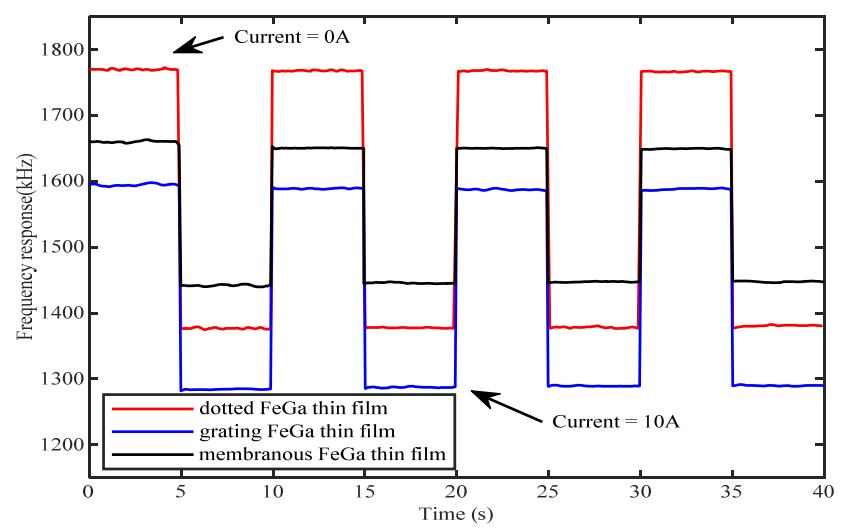

Figure 8. The repeatability of the proposed sensors.

\subsubsection{Hysteresis Measurement}

Meanwhile, the hysteresis characteristics of the FeGa thin film coated SAW devices were evaluated as shown in Figure 9a. In the measurement, the applied current increased first and then decreased with a step of $1 \mathrm{~A}$, and each applied current lasted for $5 \mathrm{~s}$. Usually, the hysteresis error is defined by

$$
\delta_{H}=\max \left(y_{u i}-y_{d i}\right) /\left(2 y_{F S}\right) \times 100 \%
$$

where the $y_{u i}$ and $y_{d i}$ are the response at the same current input when the current increases and decreases, $y_{F S}$ is the full-scale output of the current sensor. Hence, the hysteresis errors of $0.81 \%, 0.92 \%, 2.57 \%$ were calculated from the sensing devices coated with dotted, grating, and membranous FeGa thin film, respectively. Obviously, the excellent symmetry in sensor response was observed from the dotted FeGa thin-film device. Dotted pattern releases the coercivity well and enhances the magnetostrictive strain, therefore lowering the hysteresis error significantly. This can also be demonstrated by measuring the hysteresis loops of the FeGa thin film with various patterns, as shown in Figure 9b. It indicates that lower coercivity of $61.23 \mathrm{Oe}(\mathrm{Hcd})$ was observed from the dotted pattern over the grating $(\mathrm{Hcg}=72.85 \mathrm{Oe})$ and the membranous pattern $(\mathrm{Hcm}=100.17 \mathrm{Oe})$. Hence, it is reasonable to 
be concluded that the dotted pattern can not only improve the detection sensitivity but also suppress the hysteresis effect effectively in magnetostrictive thin film.
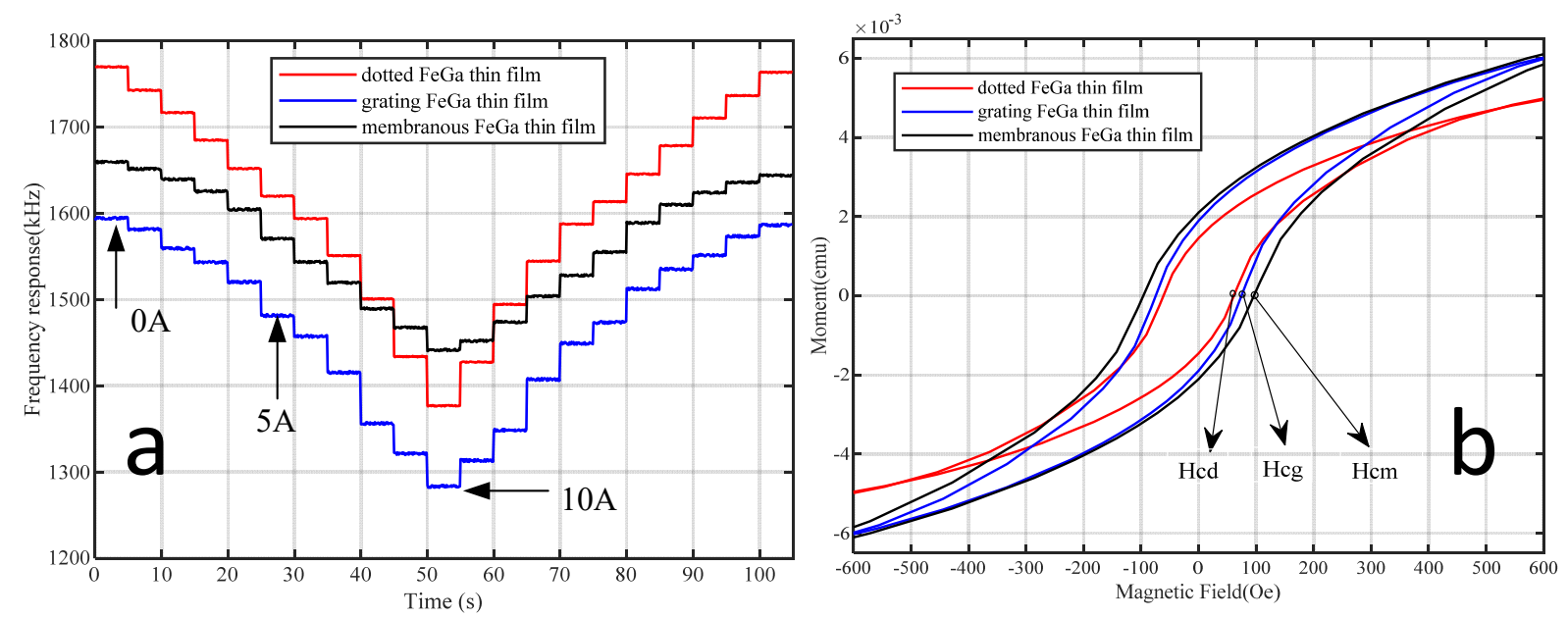

Figure 9. (a) The hysteresis of the proposed sensors; and (b) the hysteresis loop of patterned FeGa thin film.

Table 1 concludes the sensing performance of the proposed sensors with various $\mathrm{FeGa}$ film patterns. Obviously, the dot-patterned FeGa film coated sensing device features larger sensitivity and lower hysteresis error. Table 2 offers the sensing performance comparison of the proposed sensor in this work with the existing sensor prototype with similar structures. It is obvious that larger sensitivity was achieved from our work, and compared with the sensors coated with FeCo thin film [30], the sensitivity of the proposed sensors increases by $\sim 77.1 \%$. The main reason is that the FeGa thin film has a larger magnetostriction coefficient than FeCo thin film $[22,23]$.

Table 1. The sensing performance of the proposed sensors in this work.

\begin{tabular}{cccc}
\hline SAW Sensing Devices & Magnetosrictive Film & $\begin{array}{c}\text { Sensitivity } \\
\text { (kHz/A) }\end{array}$ & Hysteresis Error \\
\hline membranous -film & $\mathrm{FeGa}$ & 23.1 & $2.57 \%$ \\
grate-film & $\mathrm{FeGa}$ & 32.2 & $0.92 \%$ \\
dot-film & $\mathrm{FeGa}$ & 37.9 & $0.81 \%$ \\
\hline
\end{tabular}

Table 2. The sensing performance comparison of the proposed sensor in this work with the existing sensor prototype with similar structures.

\begin{tabular}{ccccc}
\hline Year & Material & Frequency & Sensitivity & Reference \\
\hline 2017 & FeNi & $150 \mathrm{MHz}$ & $10.7 \mathrm{kHz} / \mathrm{A}(5.35 \mathrm{kHz} / \mathrm{mT})$ & {$[11]$} \\
2020 & FeCo & $150 \mathrm{MHz}$ & $10.7 \mathrm{kHz} / \mathrm{mT}$ & {$[30]$} \\
2021 & PVA bound Fe & $433 \mathrm{MHz}$ & $678 \mathrm{kHz} / 120 \mathrm{mT}$ & {$[14]$} \\
& FeGa & $150 \mathrm{MHz}$ & $37.9 \mathrm{kHz} / \mathrm{A}(18.95 \mathrm{kHz} / \mathrm{mT})$ & our work \\
\hline
\end{tabular}

\subsubsection{Fatigue Characteristics}

Magnetostrictive strain usually leads to fatigue and aging of magnetic-sensing films. Thus, the fatigue characteristics of the FeGa thin film were investigated by cycle testing of the sensitivity of the developed sensing devices. The number of cycles was set to 100 . In each cycle test, the current of $0 \sim 10 \mathrm{~A}$ was applied to the sensing device coated with various FeGa thin-film patterns to obtain the detection sensitivity. The cycle interval was set to $10 \mathrm{~min}$. Table 3 shows the statistical analysis of the relationship between the sensor sensitivity decrease rate and the number of cycle runs. It can be seen that the sensitivity of sensors with different patterns decreased slightly with the increasing cycle runs. However, the performance decline was less than $4 \%$ after 100 cycle runs, which is 
acceptable. Obviously, thanks to the good ductility and strong impact resistance of FeGa thin film itself, excellent long-time stability and weak fatigue were observed (Figure 10a), which was far better than that of the FeCo thin film [30]. Moreover, The SEM views of the FeGa thin film after 0, 50, 100 cycle testing runs are depicted in Figure 10b-d. It can be seen that the unloaded FeGa thin film was perfectly uniform. Although a few cracks appeared after 100 runs, most areas of the film remained uniform. It means excellent stability was achieved from the FeGa thin film coated sensing device in long-term runs.

Table 3. The sensitivity decrease rate after long-term runs.

\begin{tabular}{|c|c|c|c|}
\hline \multirow{2}{*}{ Devices } & \multicolumn{3}{|c|}{ Sensitivity Decrease Rate after Long-Term Runs } \\
\hline & 0 & 50 & 100 \\
\hline membranous-film & 0 & $2.26 \%$ & $3.6 \%$ \\
\hline grate-film & 0 & $2.23 \%$ & $3.2 \%$ \\
\hline dot-film & 0 & $2.93 \%$ & $3.91 \%$ \\
\hline
\end{tabular}
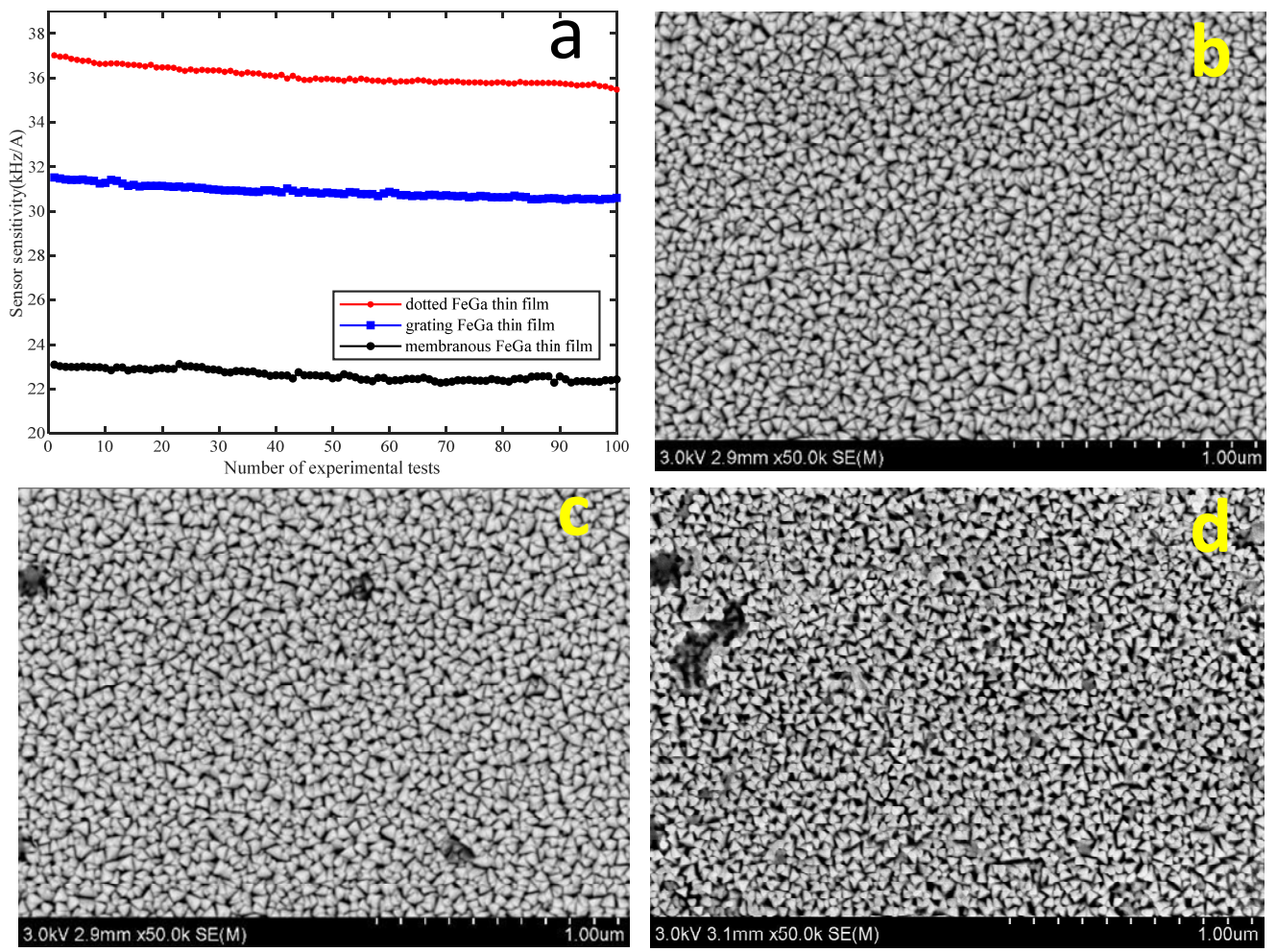

Figure 10. (a) The fatigue measurement of developed SAW sensing devices, and the surface morphologies of the FeGa thin film in different cyclic testing runs of (b) 0, (c) 50, and (d) 100.

\section{Conclusions}

The SAW current sensors with different patterns of FeGa thin films were discussed. The layered media model $\left(\mathrm{FeGa} / \mathrm{SiO}_{2} / \mathrm{LiNbO}_{3}\right)$ was established to reveal the interaction between FeGa thin film and the applied external current, and the sensitivity of the sensor was simulated. The SAW devices with various FeGa thin-film patterns (dot, grating, and membranous) were designed and fabricated to improve the detection sensitivity and lower the hysteresis error. The experimental results show that the dotted FeGa thin film coated sensing devices can achieve large current sensitivity $(37.9 \mathrm{kHz} / \mathrm{A})$, low hysteresis error $(0.81 \%)$, good repeatability, and weak fatigue, which is the result of enlarged magnetostrictive properties and reduced coercivity in the dot-film structure. In addition, compared with the existing sensor prototypes with similar structures, our proposed sensors perform far better. Those results indicate that the FeGa thin film with grating and dotted patterns can be 
used to enhance the performance of the SAW currents sensors. Hence, the dotted FeGa thin film coated SAW current sensors have broad application prospects in the fields of current detection. The obtained results provide very encouraging results for the development of wireless passive current sensors. Meanwhile, the structure of FeGa thin film with a dotted pattern can provide a new idea for the thin-film pattern design of wireless passive current sensors.

Author Contributions: Y.S.: Conceptualization, Writing-original draft, Methodology, Experiments. Y.J.: Methodology. Y.Z.: Software. L.C.: Investigation. Y.L.: Methodology. W.W.: Conceptualization, Writing-review and editing, Funding acquisition. All authors have read and agreed to the published version of the manuscript.

Funding: This research was funded by the National Natural Science Foundation of China (No.11774381, U1837209 and 52072184) and the National Key Research and Development Program (2020YFB1506203).

Institutional Review Board Statement: Not applicable.

Informed Consent Statement: Not applicable.

Data Availability Statement: Data is contained within the article.

Conflicts of Interest: The authors declare no conflict of interest.

\section{References}

1. Wang, W.; Jia, Y.; Liu, X.; Liang, Y.; Xue, X.; Du, Z. Enhanced Sensitivity of Temperature-Compensated SAW-Based Current Sensor Using the Magnetostrictive Effect. Smart Mater. Struct. 2016, 26, 025008. [CrossRef]

2. Ganguly, A.K.; Davis, K.L.; Webb, D.C.; Vittoria, C. Magnetoelastic Surface Waves in a Magnetic Film-Piezoelectric Substrate Configuration. J. Appl. Phys. 1976, 47, 2696-2704. [CrossRef]

3. Elhosni, M.; Elmazria, O.; Petit-Watelot, S.; Bouvot, L.; Zhgoon, S.; Talbi, A.; Hehn, M.; Aissa, K.A.; Hage-Ali, S.; Lacour, D.; et al. Magnetic Field SAW Sensors Based on Magnetostrictive-Piezoelectric Layered Structures: FEM Modeling and Experimental Validation. Sens. Actuators A Phys. 2016, 240, 41-49. [CrossRef]

4. Kittmann, A.; Durdaut, P.; Zabel, S.; Reermann, J.; Schmalz, J.; Spetzler, B.; Meyners, D.; Sun, N.X.; McCord, J.; Gerken, M.; et al. Wide Band Low Noise Love Wave Magnetic Field Sensor System. Sci. Rep. 2018, 8, 278. [CrossRef]

5. Steindl, R.; Hausleitner, C.; Pohl, A.; Hauser, H.; Nicolics, J. Passive Wirelessly Requestable Sensors for Magnetic Field Measurements. Sens. Actuators A Phys. 2000, 85, 169-174. [CrossRef]

6. Reindl, L.; Scholl, G.; Ostertag, T.; Ruppel, C.C.W.; Bulst, W.-E.; Seifert, F. SAW Devices as Wireless Passive Sensors. In Proceedings of the 1996 IEEE Ultrasonics Symposium, Proceedings, San Antonio, TX, USA, 3-6 November 1996; Volume 1, pp. 363-367. [CrossRef]

7. Pohl, A. A Review of Wireless SAW Sensors. IEEE Trans. Ultrason. Ferroelectr. Freq. Control 2000, 47, 317-332. [CrossRef]

8. Wang, W.; Jia, Y.; Xue, X.; Liang, Y.; Du, Z. Grating-Patterned FeCo Coated Surface Acoustic Wave Device for Sensing Magnetic Field. AIP Adv. 2018, 8, 15134. [CrossRef]

9. Wang, W.; Jia, Y.; Xue, X.; Liang, Y.; Du, Z. Magnetostrictive Effect in Micro-Dotted FeCo Film Coated Surface Acoustic Wave Devices. Smart Mater. Struct. 2018, 27, 105040. [CrossRef]

10. Ganguly, A.K.; Davis, K.L.; Webb, D.C.; Vittoria, C.; Forester, D.W. Magnetically Tuned Surface-Acoustic-Wave Phase Shifter. Electron. Lett. 1975, 11, 610-611. [CrossRef]

11. Tong, J.; Jia, Y.; Wang, W.; Wang, Y.; Wang, S.; Liu, X.; Lei, Y. Development of a Magnetostrictive FeNi Coated Surface Acoustic Wave Current Sensor. Appl. Sci. 2017, 7, 755. [CrossRef]

12. Kadota, M.; Ito, S. Sensitivity of Surface Acoustic Wave Magnetic Sensors Composed of Various Ni Electrode Structures. Jpn. J. Appl. Phys. 2012, 51, 07GC21. [CrossRef]

13. Zhou, H.; Talbi, A.; Tiercelin, N.; Bou Matar, O. Multilayer Magnetostrictive Structure Based Surface Acoustic Wave Devices. Appl. Phys. Lett. 2014, 104, 114101. [CrossRef]

14. Rajput, P.; Kumar, J.; Nimal, A.T. A Simple and Novel SAW Magnetic Sensor with PVA Bound Magnetostrictive Nanopowder Film. Sens. Actuators A Phys. 2021, 331, 112926. [CrossRef]

15. Dong, S.; Li, J.-F; Viehland, D. Ultrahigh Magnetic Field Sensitivity in Laminates of TERFENOL-D and $\mathrm{Pb}\left(\mathrm{Mg}_{1 / 3} \mathrm{Nb}_{2 / 3}\right) \mathrm{O}_{3}-$ $\mathrm{PbTiO}_{3}$ Crystals. Appl. Phys. Lett. 2003, 83, 2265-2267. [CrossRef]

16. Schell, V.; Müller, C.; Durdaut, P.; Kittmann, A.; Thormählen, L.; Lofink, F.; Meyners, D.; Höft, M.; McCord, J.; Quandt, E. Magnetic Anisotropy Controlled FeCoSiB Thin Films for Surface Acoustic Wave Magnetic Field Sensors. Appl. Phys. Lett. 2020, 116, 73503. [CrossRef]

17. Nan, T.; Hui, Y.; Rinaldi, M.; Sun, N.X. Self-Biased 215MHz Magnetoelectric NEMS Resonator for Ultra-Sensitive DC Magnetic Field Detection. Sci. Rep. 2013, 3, 1985. [CrossRef] 
18. Yang, Y.; Mishra, H.; Han, T.; Hage-Ali, S.; Hehn, M.; Elmazria, O. Sensing Mechanism of Surface Acoustic Wave Magnetic Field Sensors Based on Ferromagnetic Films. IEEE Trans. Ultrason. Ferroelectr. Freq. Control 2021, 68, 2566-2575. [CrossRef]

19. Li, W.; Dhagat, P.; Jander, A. Surface Acoustic Wave Magnetic Sensor Using Galfenol Thin Film. IEEE Trans. Magn. 2012, 48, 4100-4102. [CrossRef]

20. Ueno, T.; Summers, E.; Wun-Fogle, M.; Higuchi, T. Micro-Magnetostrictive Vibrator Using Iron-Gallium Alloy. Sens. Actuators A Phys. 2008, 148, 280-284. [CrossRef]

21. Clark, A.E.; Hathaway, K.B.; Wun-Fogle, M.; Restorff, J.B.; Lograsso, T.A.; Keppens, V.M.; Petculescu, G.; Taylor, R.A. Extraordinary Magnetoelasticity and Lattice Softening in Bcc Fe-Ga Alloys. J. Appl. Phys. 2003, 93, 8621-8623. [CrossRef]

22. Estrine, E.C.; Robbins, W.P.; Maqableh, M.M.; Stadler, B.J.H. Electrodeposition and Characterization of Magnetostrictive Galfenol (FeGa) Thin Films for Use in Microelectromechanical Systems. J. Appl. Phys. 2013, 113, 17A937. [CrossRef]

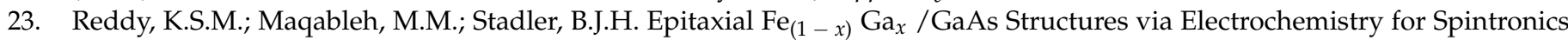
Applications. J. Appl. Phys. 2012, 111, 07E502. [CrossRef]

24. Pyati, V.P. Simplified Biot-Savart Law for Planar Circuits. IEEE Trans. Educ. 1986, E-29, 32-33. [CrossRef]

25. Wen, W.; Shitang, H.; Shunzhou, L.; Minghua, L.; Yong, P. Enhanced Sensitivity of SAW Gas Sensor Coated Molecularly Imprinted Polymer Incorporating High Frequency Stability Oscillator. Sens. Actuators B Chem. 2007, 125, 422-427. [CrossRef]

26. Ou-Yang, J.; Liu, X.; Zhou, H.; Zou, Z.; Yang, Y.; Li, J.; Zhang, Y.; Zhu, B.; Chen, S.; Yang, X. Magnetoelectric Laminate Composites: An Overview of Methods for Improving the DC and Low-Frequency Response. J. Phys. D Appl. Phys. 2018, 51, 324005. [CrossRef]

27. Zhai, J.; Xing, Z.; Dong, S.; Li, J.; Viehland, D. Magnetoelectric Laminate Composites: An Overview. J. Am. Ceram. Soc. 2008, 91 , 351-358. [CrossRef]

28. Xing, Z.; Zhai, J.; Li, J.; Viehland, D. Investigation of External Noise and Its Rejection in Magnetoelectric Sensor Design. J. Appl. Phys. 2009, 106, 24512. [CrossRef]

29. Tiersten, H.F.; Sinha, B.K. A Perturbation Analysis of the Attenuation and Dispersion of Surface Waves. J. Appl. Phys. 1978, 49, 87-95. [CrossRef]

30. Jia, Y.; Wang, W.; Sun, Y.; Liu, M.; Xue, X.; Liang, Y.; Du, Z.; Luo, J. Fatigue Characteristics of Magnetostrictive Thin-Film Coated Surface Acoustic Wave Devices for Sensing Magnetic Field. IEEE Access 2020, 8, 38347-38354. [CrossRef] 